\title{
Displasia fibrosa de columna lumbar en una mujer de mediana edad. Presentación de un caso
}

\author{
Julia M. Saidman, Damián Di Memmo, Alejandro Rasumoff \\ Servicio de Diagnóstico por Imágenes, Hospital Italiano de Buenos Aires, Ciudad Autónoma de Buenos Aires, Argentina
}

\begin{abstract}
RESUMEN
La displasia fibrosa es una lesión ósea benigna caracterizada por el desarrollo anormal del tejido fibroso de disposición arremolinada, con trabéculas de hueso inmaduro no laminar. Se distinguen dos formas: monostótica y poliostótica. El compromiso de columna vertebral se asocia, con más frecuencia, a la variedad poliostótica; la forma monostótica es infrecuente. Presentamos a una mujer de 46 años que consultó por lumbocruralgia derecha de dos meses de evolución, con diagnóstico definitivo de displasia fibrosa de columna lumbar. Los estudios por imágenes mostraron una lesión monostótica lítico-quística con tabiques internos localizada en el arco posterior de la quinta vértebra lumbar. Sus características en los estudios por imágenes sugirieron un quiste óseo aneurismático, mientras que la anatomía patológica fue reveladora frente al diagnóstico final de displasia fibrosa. Los síntomas menores y la ausencia de complicaciones llevaron a indicar un tratamiento conservador. Si bien el compromiso de columna lumbar por displasia fibrosa monostótica es infrecuente, debería considerarse entre los diagnósticos diferenciales de una lesión lítica solitaria en dicha localización. No obstante, no se descarta mediante histopatología que pueda tratarse de un caso de coexistencia de displasia fibrosa y quiste óseo aneurismático o que la displasia fibrosa se haya desarrollado sobre un quiste óseo aneurismático.
\end{abstract}

Palabras clave: Displasia fibrosa; quiste óseo aneurismático; columna lumbar.

Nivel de Evidencia: IV

Fibrous dysplasia of the lumbar spine in a middle-aged woman. Case presentation

\begin{abstract}
Fibrous dysplasia (FD) is a benign skeletal disorder characterized by abnormal development of fibrous tissue in a whorled pattern and containing trabeculae of immature non-lamellar bone. FD has two forms of clinical presentations: monostotic and polyostotic. Spinal involvement is seen mostly in the polyostotic form and is very unusual in the monostotic form. We present a 46-year-old woman that complained of right low back pain with a 2-month evolution. The definitive diagnosis was FD of the lumbar spine. Imaging testing revealed a lytic-cystic monostotic lesion with internal septa located in the posterior arch of the fifth lumbar vertebra, suggestive of Aneurysmal Bone Cyst $(A B C)$. However, the anatomical pathology revealed FD as the final diagnosis. Conservative treatment was undertaken due to minimal symptoms and the absence of complications. Although monostotic FD of lumbar spine is rare, it should be taken into account among the differential diagnoses of a single osteolytic lesion. However, histopathology testing cannot rule out the coexistence of $F B$ and $A B C$ or a setting of FB secondary to an $A B C$.
\end{abstract}

Key words: Fibrous dysplasia; aneurysmal bone cyst; lumbar spine.

Level of Evidence: IV

Recibido el 13-4-2019. Aceptado luego de la evaluación el 7-11-2019 • Dra. JULIA M. SAIDMAN • julia.saidman @ hospitalitaliano.com.ar (ID

Cómo citar este artículo: Saidman JM, Di Memmo D, Rasumoff A. Displasia fibrosa de columna lumbar en una mujer de mediana edad. Presentación de un caso. Rev Asoc Argent Ortop Traumatol 2020;85(2):167-173. https://doi.org/10.15417/issn.1852-7434.2020.85.2.980 


\section{INTRODUCCIÓN}

Las lesiones tumorales de la columna vertebral más frecuentes son las metástasis óseas, el mieloma múltiple y el linfoma. ${ }^{1,2}$ Las lesiones óseas conocidas como "seudotumorales" representan un pequeño porcentaje de las afecciones óseas y comprenden un amplio rango de enfermedades de naturaleza benigna y que, a menudo, plantean un desafío diagnóstico-terapéutico para el equipo médico interdisciplinario. ${ }^{3,4}$

La displasia fibrosa (DF) es una enfermedad fibro-ósea de baja frecuencia, que se produce por un desarrollo anormal del tejido fibroso de disposición arremolinada, en el que se encuentran trabéculas de hueso inmaduro no laminar. Afecta tanto a niños como adultos, sin prevalencia de sexo, y representa el 7\% de los tumores óseos. ${ }^{1,5}$

La lesión suele ser asintomática y un hallazgo incidental, aunque puede presentarse con dolor y tumefacción, o complicaciones que abarcan desde una fractura patológica hasta la transformación sarcomatosa en un pequeño porcentaje de casos..$^{1,4,6}$

Se distinguen dos formas clínicas de presentación: monostótica o poliostótica. Sin bien comparten características radiológicas, histológicas y sitios anatómicos de localización, se observan diferencias entre ellas. ${ }^{1,4} \mathrm{El} 80 \%$ de los casos corresponde a la forma monostótica y afecta, en mayor medida, costillas, fémur, tibia, mandíbula, cráneo y húmero. ${ }^{6}$ Por otro lado, la variedad poliostótica compromete principalmente cráneo, huesos faciales, pelvis, columna vertebral, y puede relacionarse con diversos síndromes, como de McCune-Albright (anomalías neuroendocrinas y pigmentación cutánea) o de Mazabraud (mixomas intramusculares). ${ }^{4,6}$

En cuanto a las características sugestivas de DF en los estudios por imágenes, cabe destacar que se trata de lesiones con una presentación variable muy marcada. ${ }^{1,2}$

En la radiografía simple y la tomografía computarizada (TC), se pueden observar desde imágenes escleróticas con un típico fondo en "vidrio esmerilado", lesiones líticas con borde esclerótico, hasta lesiones que expanden el hueso con contenido de aspecto quístico. ${ }^{1,6}$ La resonancia magnética (RM) muestra lesiones con señal intermedia/ baja en secuencias ponderadas en T1, intermedia/alta en secuencias T2, con un realce heterogéneo tras la administración de gadolinio por vía endovenosa. ${ }^{1,6}$

Desde el punto de vista histológico, la lesión aparece típicamente bien circunscrita, compuesta por tejido fibroso uniformemente celular que contiene proliferación de células fusiformes con escasa actividad mitótica. Dispersas a través de la matriz fibrosa se encuentran las laminillas o nidos redondeados de tejido óseo sin un adelgazamiento osteoblástico significativo. ${ }^{4}$

Con respecto a la conducta terapéutica, puede optarse por un tratamiento conservador, ya que las lesiones suelen mantenerse estables a lo largo del tiempo; la radioterapia conlleva un riesgo incrementado de transformación maligna y, por ende, debería evitarse. ${ }^{4}$

Por otra parte, dentro del grupo de las lesiones óseas "seudotumorales", debemos mencionar al quiste óseo aneurismático (QOA), que representa uno de los diagnósticos diferenciales de la DF. Se trata de un cuadro con una frecuencia del 1,4-2,3\%, que se manifiesta, en gran medida, en la segunda década de la vida.

Las localizaciones anatómicas más frecuentes comprenden la metáfisis de huesos largos (fémur distal y tibia proximal) y la columna vertebral (3-20\% de los casos). ${ }^{4} \mathrm{El} \mathrm{compromiso} \mathrm{de} \mathrm{la} \mathrm{columna} \mathrm{torácica} \mathrm{es} \mathrm{más} \mathrm{frecuente} \mathrm{y}$ le siguen el de la columna lumbar y cervical. ${ }^{1,3}$ Debe tenerse en cuenta la típica afectación del arco posterior y la posibilidad de diseminación a vértebras adyacentes, disco intervertebral, costillas y partes blandas paravertebrales. ${ }^{1}$

El cuadro clínico varía según la localización y el tamaño de la lesión; la mayoría de los pacientes cursan con dolor e inflamación, en tanto que el compromiso vertebral puede provocar síntomas de compresión medular y radicular. ${ }^{1,2}$

Los estudios por imágenes revelan los hallazgos característicos. La radiografía muestra típicamente lesiones radiolúcidas, expansivas, con tabiques y de bordes bien delimitados; en tanto que, en la TC y RM, se observan niveles líquido-líquido por hemorragia con sedimentación. ${ }^{1,2,4}$ La RM es el método diagnóstico con mayor sensibilidad, permite observar lesiones bien definidas con tabiques internos con los diferentes niveles líquido-líquido, representados típicamente como áreas de aumento de señal en secuencias ponderadas en T1 debido al componente de metahemoglobina. ${ }^{1,2}$ Cabe mencionar que el hallazgo de niveles líquido-líquido es sugestivo de QOA, pero no patognomónico. ${ }^{1,2}$

En el análisis histopatológico, se observan espacios quísticos llenos de sangre, separados por estroma celular, con células gigantes similares a osteoclastos y osteoide. ${ }^{1}$ 
Las opciones terapéuticas dependen, en gran parte, de la localización y la extensión de la lesión. Si bien el curetaje y el relleno del defecto con injerto óseo han sido considerados el tratamiento estándar, se dispone de una gama de opciones terapéuticas adaptadas con el fin de reducir la morbimortalidad, ${ }^{7}$ como el uso de adyuvantes (coagulación con argón o fenol), terapias alternativas (embolización arterial selectiva preoperatoria y radioterapia neoadyuvante) y técnicas emergentes prometedoras, menos agresivas, por ejemplo, doxiciclina percutánea, bifosfonatos y denosumab.

\section{CASO CLÍNICO}

Se trata de una mujer de 46 años de edad, cuyos únicos antecedentes de relevancia son una hipoacusia bilateral, el uso de audífonos desde los 12 años y una cirugía de cataratas a los 42 años.

Consultó, en nuestra institución, por lumbociatalgia derecha de tipo punzante de dos meses de evolución, que había sido tratada, en un ámbito extrainstitucional, mediante kinesioterapia y tratamiento del dolor. La paciente negaba antecedentes de traumatismo/caída u oncológicos.

La anamnesis y el examen físico a cargo del Servicio de Ortopedia y Traumatología no revelaron otros hallazgos.

Se solicitó una TC de columna lumbosacra que mostró una formación de aspecto lítico de bordes escleróticos y delgados tabiques internos completos, expansiva y centrada en el pedículo derecho de la quinta vértebra lumbar y con extensión al cuerpo vertebral, faceta y apófisis transversa derecha. Insuflaba y adelgazaba la cortical sin componentes extraóseos y sin comprometer los forámenes de conjunción (Figura 1).

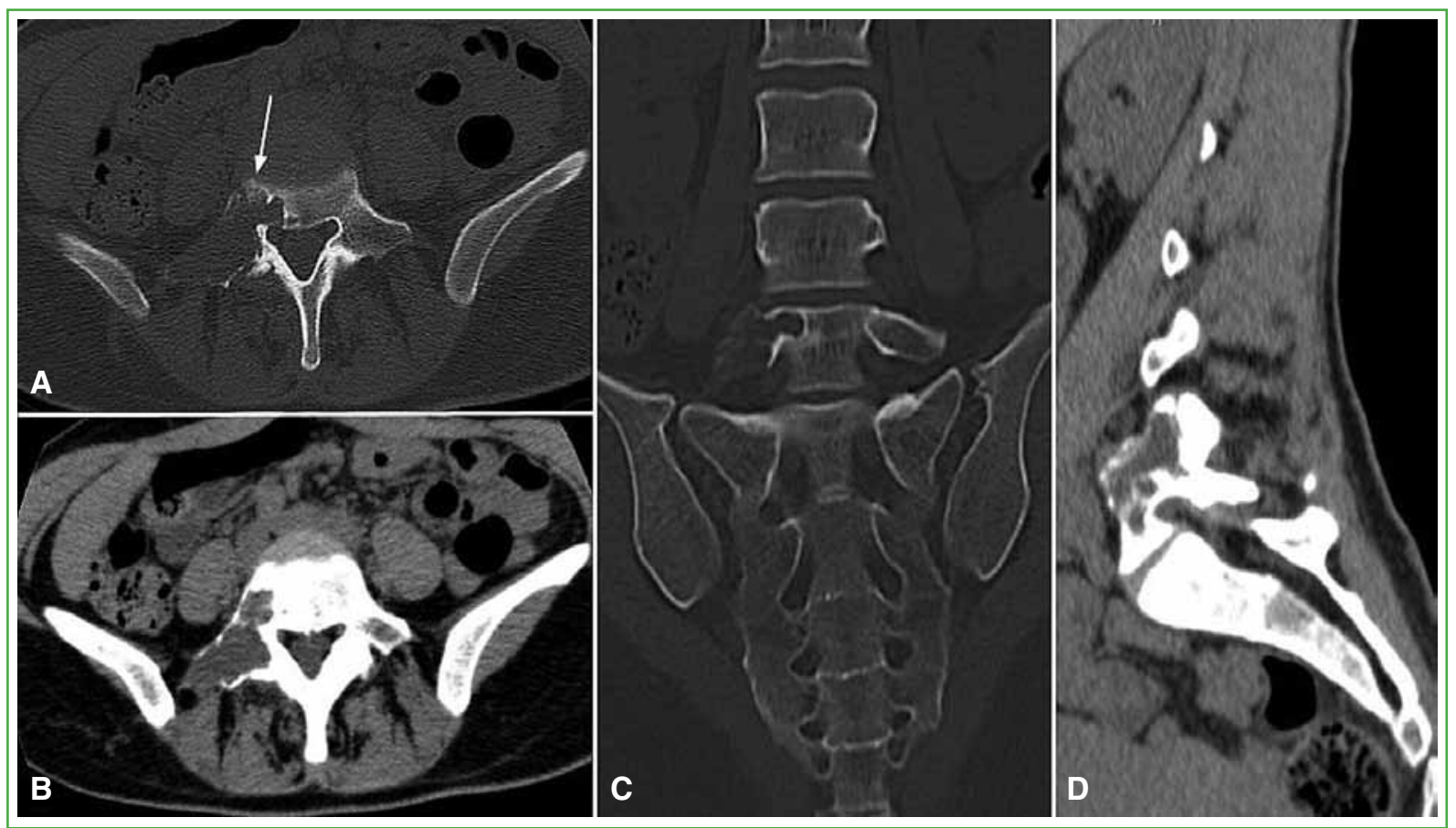

Figura 1. Tomografía computarizada. A. Ventana ósea, corte axial. B. Partes blandas, corte axial. C. Ventana ósea, corte coronal. D. Partes blandas, corte sagital. Lesión de aspecto lítico de bordes escleróticos y delgados tabiques internos completos, expansiva que adelgaza la cortical, centrada en el pedículo derecho de L5 y con extensión al cuerpo vertebral, faceta y apófisis transversa derecha. Presenta una pequeña área en "vidrio esmerilado" (flecha). 
En la RM de columna lumbosacra con contraste endovenoso, se identificó la misma lesión expansiva de 24 mm x $33 \mathrm{~mm}$ x $16 \mathrm{~mm}$, de contenido líquido, con tabiques internos y adelgazamiento de la cortical ósea, centrada en el pedículo derecho de L5 y con extensión al cuerpo vertebral, faceta y apófisis transversa derecha (Figura 2).

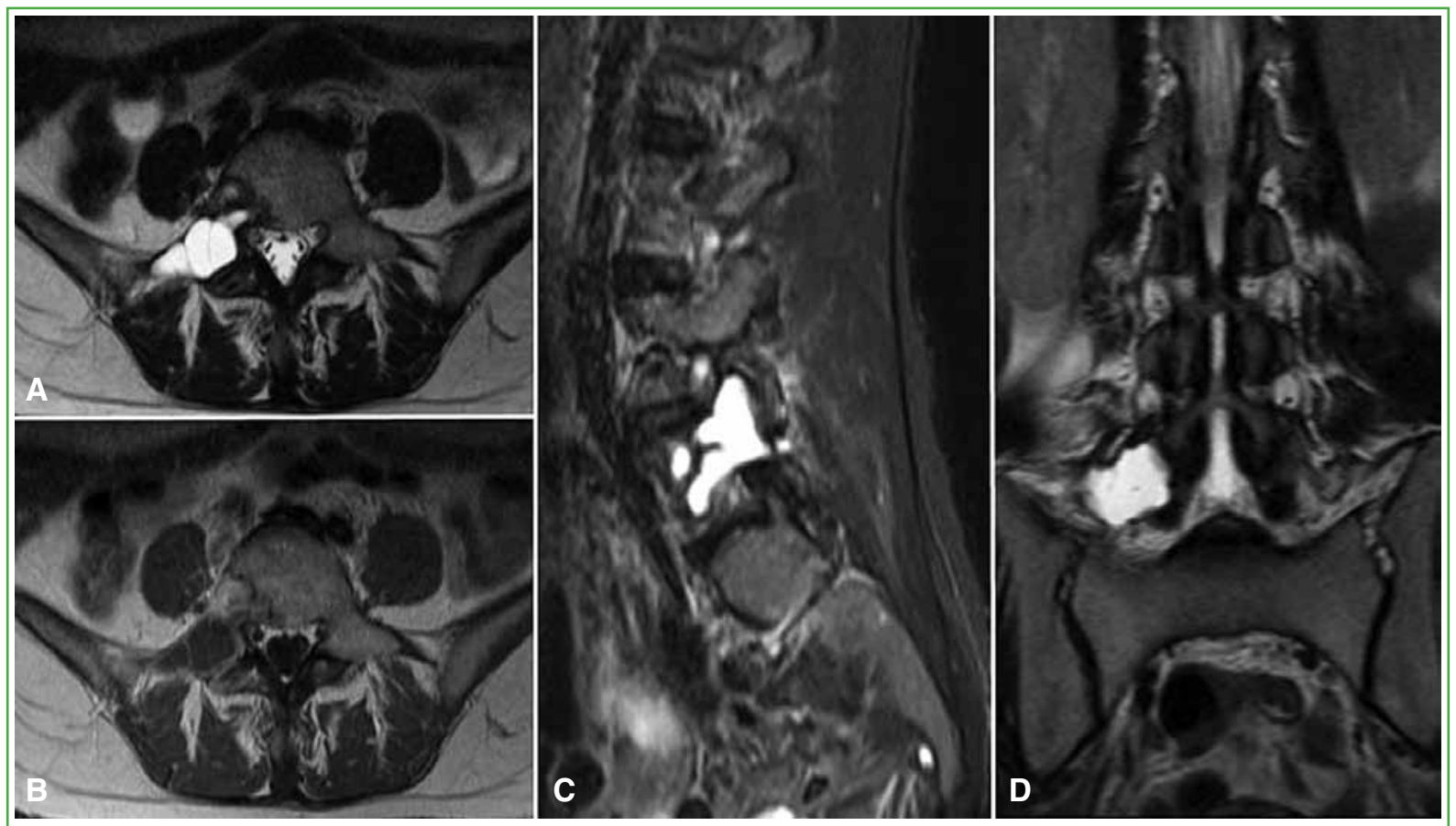

Figura 2. Resonancia magnética. A. T2, corte axial. B. T1, corte axial. C. STIR, corte sagital. D. T2, corte coronal. Lesión de intensidad de señal líquida (hiperintensa en secuencia T2 y STIR e hipointensa en secuencia T1) con tabiques internos, expansiva que genera adelgazamiento de la cortical ósea, centrada en el pedículo derecho de L5 y con extensión al cuerpo vertebral, faceta y apófisis transversa derecha.

Las características de las imágenes por TC y RM, y la localización de la lesión llevaron a interpretar el cuadro como un QOA.

Se realizó una punción-biopsia bajo guía tomográfica mediante un acceso posterolateral derecho; se obtuvieron $10 \mathrm{~cm}^{3}$ de material serohemático en jeringa para estudio citológico y un pequeño taco óseo para estudio histológico. No hubo complicaciones durante el procedimiento. En el estudio citológico mediante centrifugación, se observó un extendido con abundantes hematíes y elementos sanguíneos, hueso con material hemático hipocelular. En el estudio histopatológico, se visualizaron trabéculas de hueso reticular, sin ribete osteoblástico prominente, en relación con los espacios nodulares fibrosos, lo que llevó al diagnóstico final de DF (Figura 3).

Sobre la base del diagnóstico definitivo de DF, se optó por adoptar una conducta expectante con seguimiento periódico clínico y por imágenes. La paciente continúa en evaluación tras 14 meses desde el diagnóstico, con adecuado manejo sintomático y sin complicaciones asociadas. 


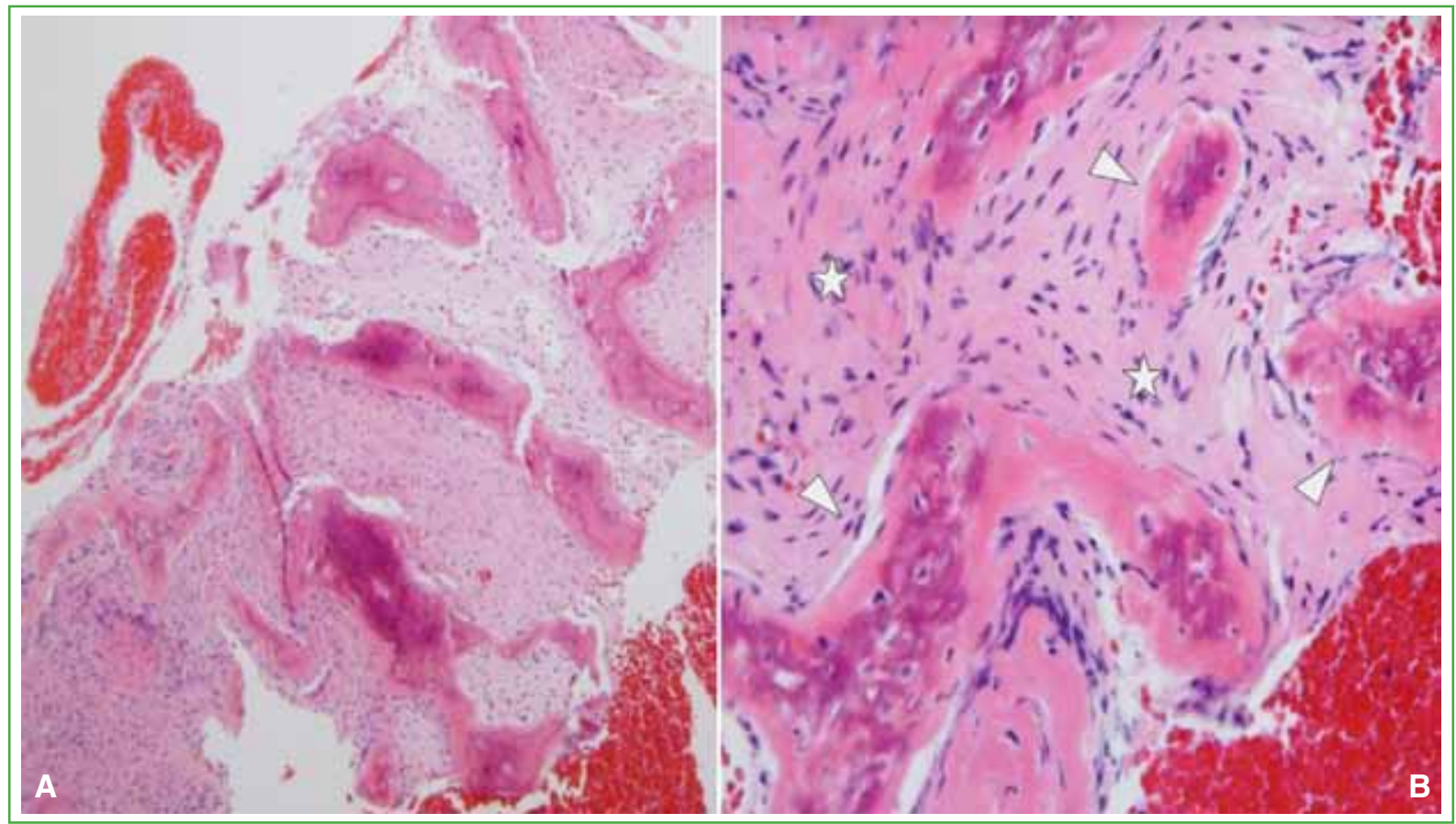

Figura 3. Anatomía patológica. A. 40x. B. 100x. Los cortes histológicos muestran trabéculas de hueso reticular sin ribete osteoblástico prominente (cabezas de flecha), en relación con los espacios medulares fibrosos $(*)$, hallazgos compatibles con displasia fibrosa.

\section{DISCUSIÓN}

La DF y el QOA se encuadran dentro del grupo de las lesiones óseas seudotumorales de naturaleza benigna. . $^{3,4,8}$ Sus formas de presentación, sus características en los estudios por imágenes y su localización pueden tener una marcada variabilidad y, al mismo tiempo, pueden compartir características en los estudios por imágenes; por lo tanto, debe plantearse el diagnóstico diferencial entre estas dos entidades y el resto de las enfermedades óseas tumorales hasta obtener un diagnóstico anatomopatológico definitivo. , $^{3,10,11}$

El QOA puede tratarse de una lesión primaria o secundaria a enfermedades óseas que quistifican (un tercio de los casos), ${ }^{1}$ uno de las más frecuentes es el tumor de células gigantes y le siguen el osteoblastoma, el angioma, el condroblastoma, la DF y el granuloma eosinofílico. ${ }^{1}$ En consecuencia, debe tenerse en cuenta la posibilidad de que una lesión como la DF pueda quistificarse y dar lugar a la formación de un QOA. ${ }^{1}$

Un tercer escenario posible es la coexistencia de componentes de DF y QOA en una misma lesión, algo extremadamente infrecuente y reportado en muy pocos casos. Buraczewski y Dabska comunicaron dos casos de coexistencia de QOA y DF en un total de 25, uno de ellos localizado en la tercera costilla posterior y el otro, en el ángulo derecho de la mandíbula. ${ }^{9}$ Martinez y Sissons estudiaron a 42 pacientes con DF y hallaron componente de QOA adicional en un caso de compromiso de la quinta costilla; ${ }^{12}$ en tanto que Lee y cols. publicaron un caso similar en la región frontoparietal derecha. ${ }^{11}$

La DF, rara vez, afecta la columna vertebral y, en este caso, es más frecuente en el contexto de una forma poliostótica. En nuestro caso, se trató de una DF monostótica con compromiso espinal, lo que configura una entidad muy poco frecuente. Solo 13 de los casos de DF con compromiso vertebral correspondían a la columna lumbar, 5,13 lo que refuerza la singularidad del caso presentado.

En el diagnóstico diferencial de lesiones óseas monostóticas del arco vertebral posterior, deben incluirse el osteoma osteoide, el osteoblastoma, el osteocondroma y el QOA. ${ }^{1}$ El osteoma osteoide se presenta como una lesión esclerótica con un nido central de menor densidad y cursa con dolor nocturno; el osteoblastoma puede ser indistinguible del osteoma osteoide y, en general, presenta un nido central de mayor tamaño; en tanto que el osteocondroma suele ser una lesión excéntrica sésil-pediculada. Como excepciones deben considerarse las lesiones malignas: condrosarcoma, osteosarcoma y sarcoma de Ewing. ${ }^{1}$ 
La principal limitación de nuestro análisis es que no se puede descartar la posibilidad de que exista un componente de QOA intralesional, dado que la toma de biopsia se realizó por vía posterolateral de solo un segmento periférico de la lesión y, por ende, que se trate de un caso de coexistencia DF-QOA. Por otro lado, tampoco se puede excluir que el curso de la lesión haya sido la transformación de QOA a DF en un lapso evolutivo, debido a la falta de consultas y de estudios previos.

\section{CONCLUSIONES}

Comunicamos un caso de una lesión monostótica del arco posterior de la columna lumbar. Ante los datos que el equipo médico obtuvo para llegar al diagnóstico presuntivo -cuadro clínico y estudios por imágenes- se planteó una fuerte sospecha de que se tratase de una presentación clásica del QOA. Sin embargo, el diagnóstico final anatomopatológico fue revelador frente al hallazgo de DF. Si bien debemos tener en cuenta la epidemiología y los diagnósticos diferenciales más frecuentes ante una lesión del arco vertebral posterior, no debemos perder de vista la posibilidad de que se trate de lesiones con localización y presentación inusual.

Conflicto de intereses: Los autores no declaran conflictos de intereses.

ORCID de D. Di Memmo: https://orcid.org/0000-0002-1675-964X

ORCID de A. Rasumoff: https://orcid.org/0000-0002-7505-501X

\section{BIBLIOGRAFÍA}

1. Rodallec M, Feydy A, Larousserie F, Anract P, Campagna R, Babinet A, et al. Diagnostic imaging of solitary tumors of the spine: what to do and say. Radiographics 2008;28(4):1019-41. https://dx.doi.org/10.1148/rg.284075156

2. Murphey M, Andrews C, Flemming D, Temple T, Smith S, Smirniotopoulos J. From the archives of the AFIP. Primary tumors of the spine: radiologic pathologic correlation. Radiographics 1996;16(5):1131-58. https://dx.doi.org/10.1148/radiographics.16.5.8888395

3. Miller T. Bone tumors and tumor-like conditions: analysis with conventional radiography. Radiology 2008;246(3):662-74. https://doi.org/10.1148/radiol.2463061038

4. Remotti F, Feldman F. Nonneoplastic lesions that simulate primary tumors of bone. Arch Pathol Lab Med 2012;136(7):772-88. https://doi.org/10.5858/arpa.2011-0557-RA

5. Chow LTC, Griffith J, Chow WH, Kumta SM. Monostotic fibrous dysplasia of the spine: report of a case involving the lumbar transverse process and review of the literature. Arch Orthop Trauma Surg 2000;120(7):460-4. https://doi.org/10.1007/p100013774

6. Fitzpatrick K, Taljanovic M, Speer D, Graham A, Jacobson J, Barnes J, et al. Imaging findings of fibrous dysplasia with histopathologic and intraoperative correlation. AJR Am J Roentgenol 2004;182(6):1389-98. https://doi.org/10.2214/ajr.182.6.1821389

7. Park H, Yang S, Sheppard W, Hegde V, Zoller S, Nelson S, et al. Current management of aneurysmal bone cysts. Curr Rev Musculoskelet Med 2016;9(4):435-44. https://doi.org/10.1007/s12178-016-9371-6

8. Munday T, Jobnson M, Whayes C, Thompson E, Smokei W. Musculoskeletal causes of spinal axis compromise: beyond the usual suspects. Radiographics 1994;14(6):1225-45. https://doi.org/10.1148/radiographics.14.6.7855338

9. Buraczewski J, Dabska M. Pathogenesis of aneurysmal bone cyst. Relationship between the aneurysmal bone cyst and fibrous dysplasia of bone. Cancer 1971;28(3):597-604. https://doi.org/10.1002/1097-0142(197109)28:3<597::aid-cncr2820280311>3.0.co;2-i

10. Kransdorf M, Sweet D. Aneurysmal bone cyst: concept, controversy, clinical presentation, and imaging. AJR Am J Roentgenol 1995;164(3):573-80. https://doi.org/10.2214/ajr.164.3.7863874 
11. Lee JW, Kim JH, Han SH, Kang HI. Fibrous dysplasia with aneurysmal bone cyst presenting as painful solitary skull lesion. J Korean Neurosurg Soc 2010;48(6):551-4. https://doi.org/10.3340/jkns.2010.48.6.551

12. Martinez V, Sissons HA. Aneurysmal bone cyst. A review of 123 cases including primary lesions and those secondary to other bone pathology. Cancer 1988;61(11):2291-2304.

https://doi.org/10.1002/1097-0142(19880601)61:11<2291::aid-cncr2820611125>3.0.co;2-v

13. Asazuma T, Sato M, Masuoka K, Yasuoka H, Tsuji T, Aida S. Monostotic fibrous dysplasia of the lumbar spine. Case report and review of the literature. J Spinal Disord Tech 2005;18(6):535-8.

https://doi.org/10.1097/01.bsd.0000127703.75711.e7 